\title{
A New Era in Systemic Therapy for Hepatocellular Carcinoma: Atezolizumab plus Bevacizumab Combination Therapy
}

\author{
Masatoshi Kudo \\ Department of Gastroenterology and Hepatology, Kindai University Faculty of Medicine, \\ Osaka-Sayama, Japan
}

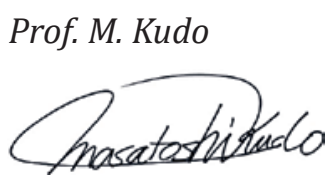

Editor Liver Cancer

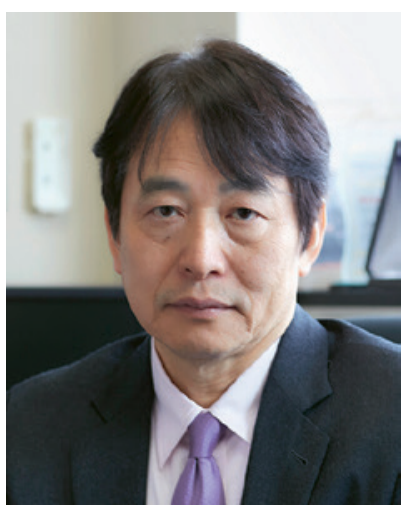

\section{Scientific Rationale for Combination Immunotherapy with PD-1/PD-L1 Antibody plus Anti-VEGF/Molecular Targeted Agent}

High levels of vascular endothelial growth factor (VEGF) released from hypoxic tumor cells and the vascular endothelium induce angiogenesis, thereby contributing to proliferation, invasion, and metastasis of cancer cells. In addition, VEGF promotes the recruitment and proliferation of various types of immunosuppressive cells, such as regulatory T-cells (Tregs), and the release of pro-tumor cytokines from these cells. VEGF also increases the recruitment of tumorassociated macrophages (TAMs), which increases the M2 phenotype activity at the tumor site. Recruited Tregs and TAMs also contribute to tumor proliferation by releasing VEGF and angiopoietin 2. Similarly, VEGF induces myeloid-derived suppressor cells (MDSCs), which also release VEGF. Meanwhile, VEGF has a suppressive effect on dendritic cells (DCs). It decreases the number of DCs and inhibits their maturation, thereby suppressing the antigen-presenting ability of DCs in the priming phase. Therefore, despite their presence, neoantigens are not presented on DCs, and the proliferation and activation of CD8-positive cells are impaired. In addition, cells upregu- 


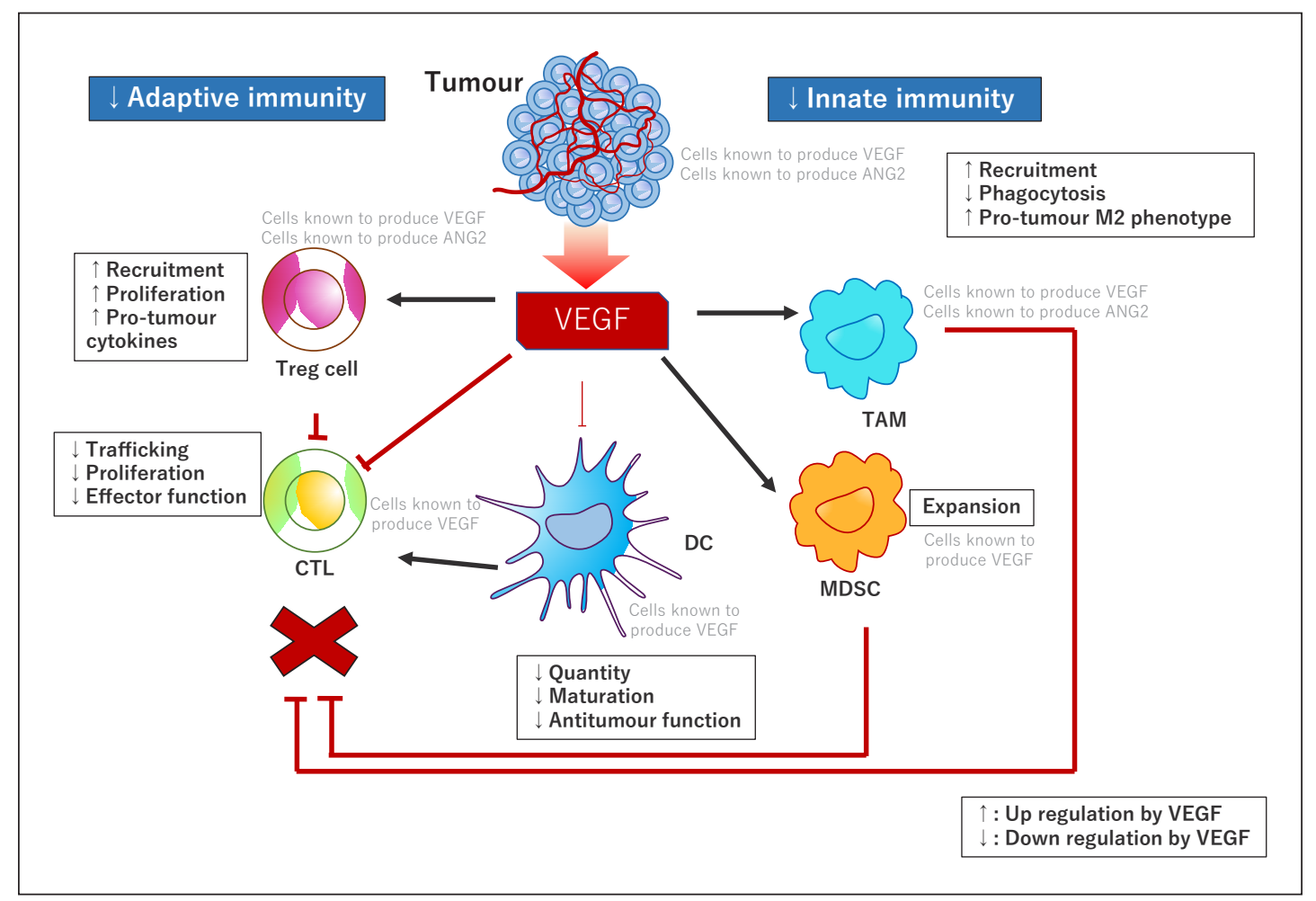

Fig. 1. Direct effects of angiogenic factors on various immune cells [modified from 1].

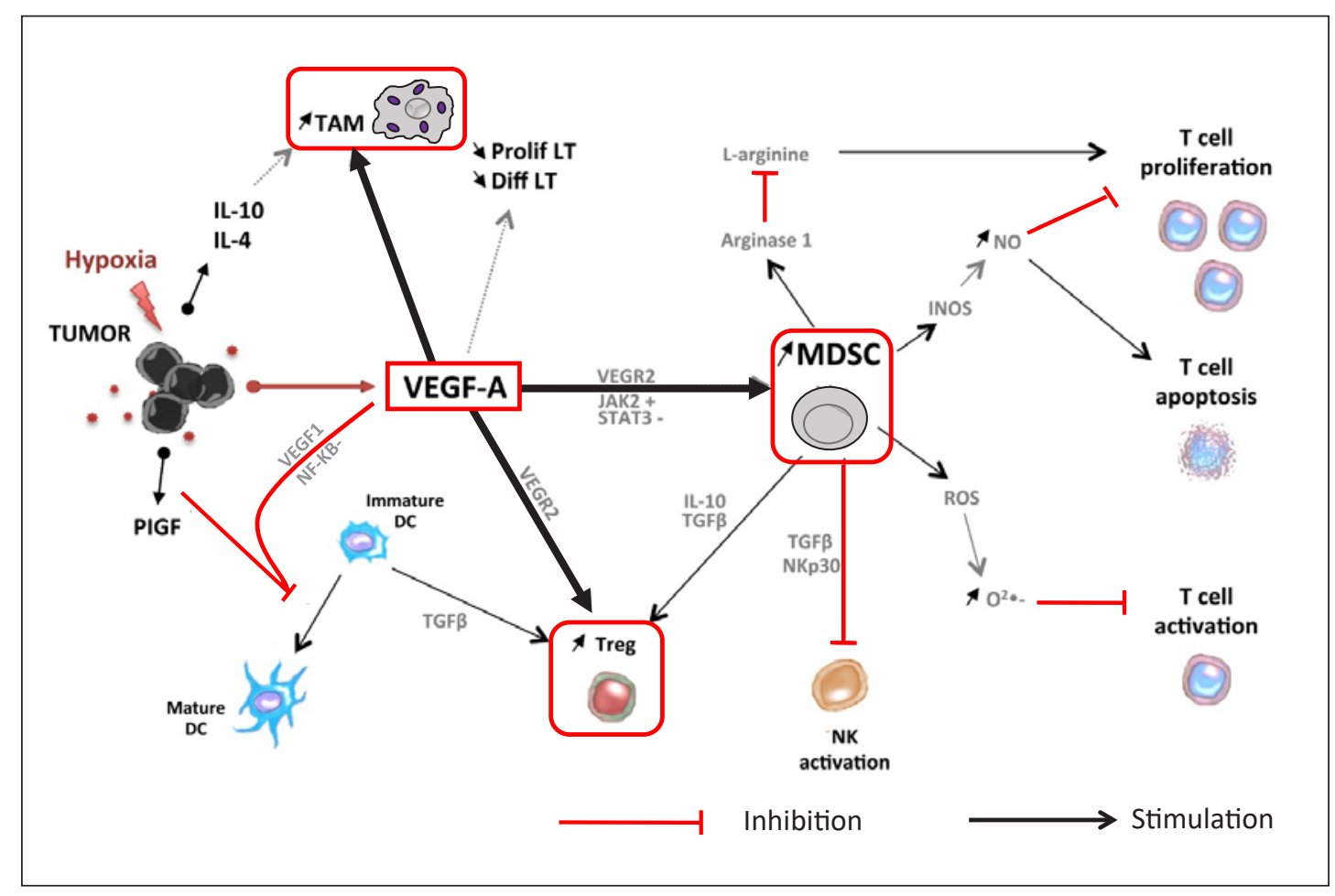

Fig. 2. Immunosuppressive microenvironment induced by VEGF [modified from 3]. 


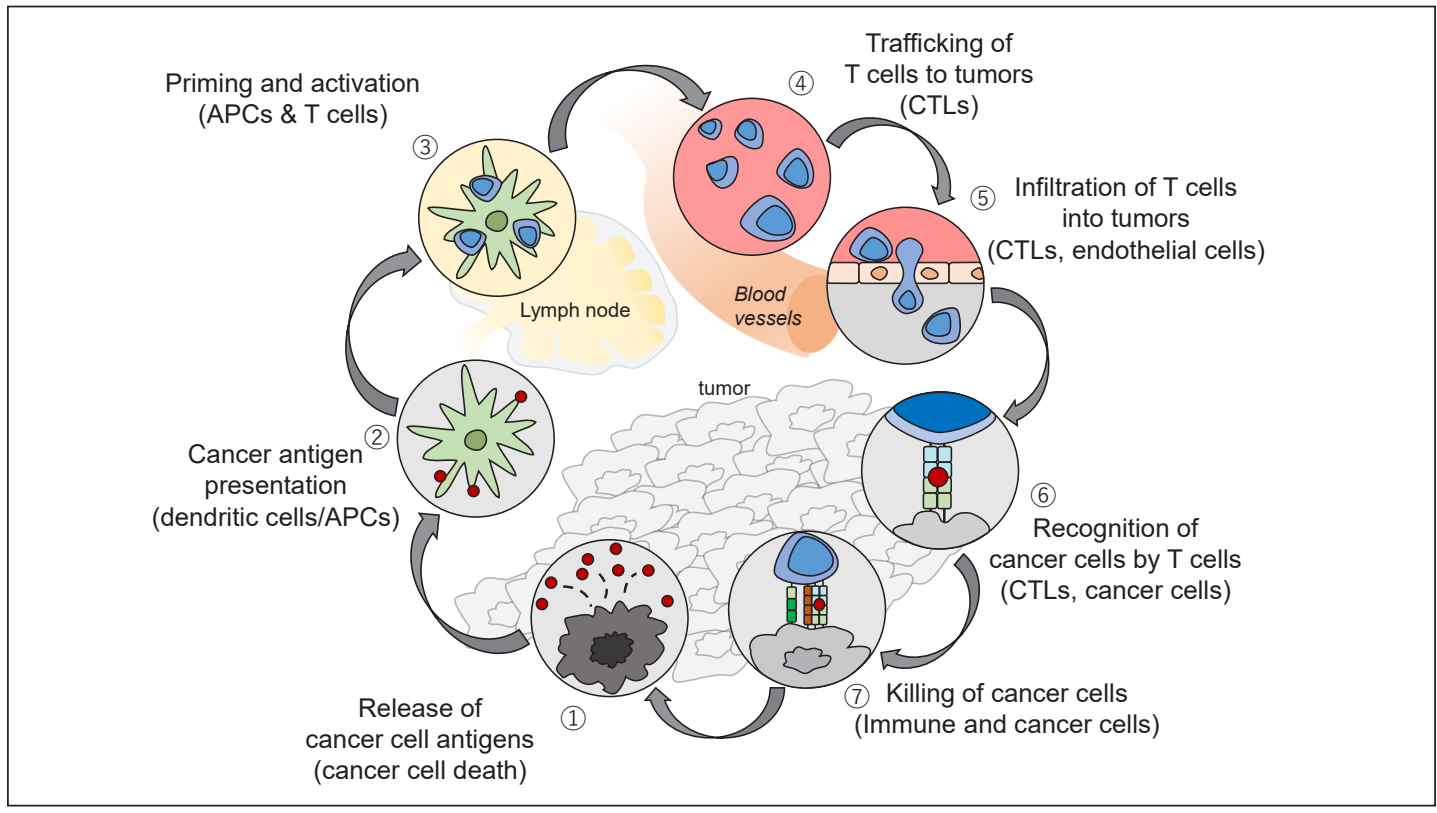

Fig. 3. The cancer-immunity cycle [modified from 4].

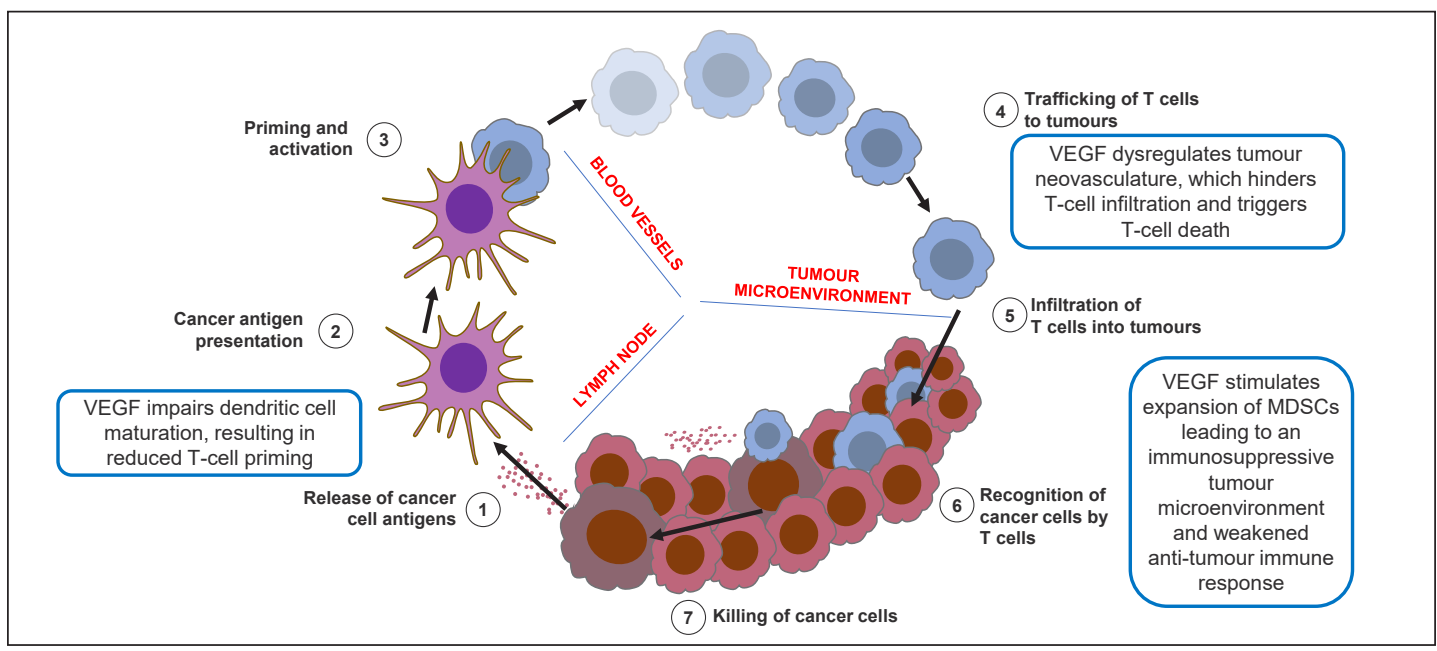

Fig. 4. VEGF influences multiple steps, leading to immune escape [modified from 4-8, 10-21, 25].

lated by VEGF (Tregs, TAMs, and MDSCs) suppress the proliferation and effector function of CD8-positive cells. Although CD8-positive cells are activated via antigen presentation, VEGFinduced abnormal tumor angiogenesis inhibits trafficking and infiltration of activated CD8-positive cells to the cancer cells. This leads to the formation of a tumor microenvironment wherein T-cells in the effector phase are disarmed. Furthermore, the release of immunosuppressive cytokines (IL-10, TGF- $\beta$ ) from these immunosuppressive cells (Tregs, MDSCs, and TAMs) augments the microenvironment and allows immune escape, thereby inhibiting DC maturation and activation, natural killer cell activation, and T-cell activation and proliferation, and establishing an immunosuppressive microenvironment [1-24] (Fig. 1, 2). The cancer-immunity cycle is a sequence of antitumor immune responses initiated upon presentation by DCs of neoan- 


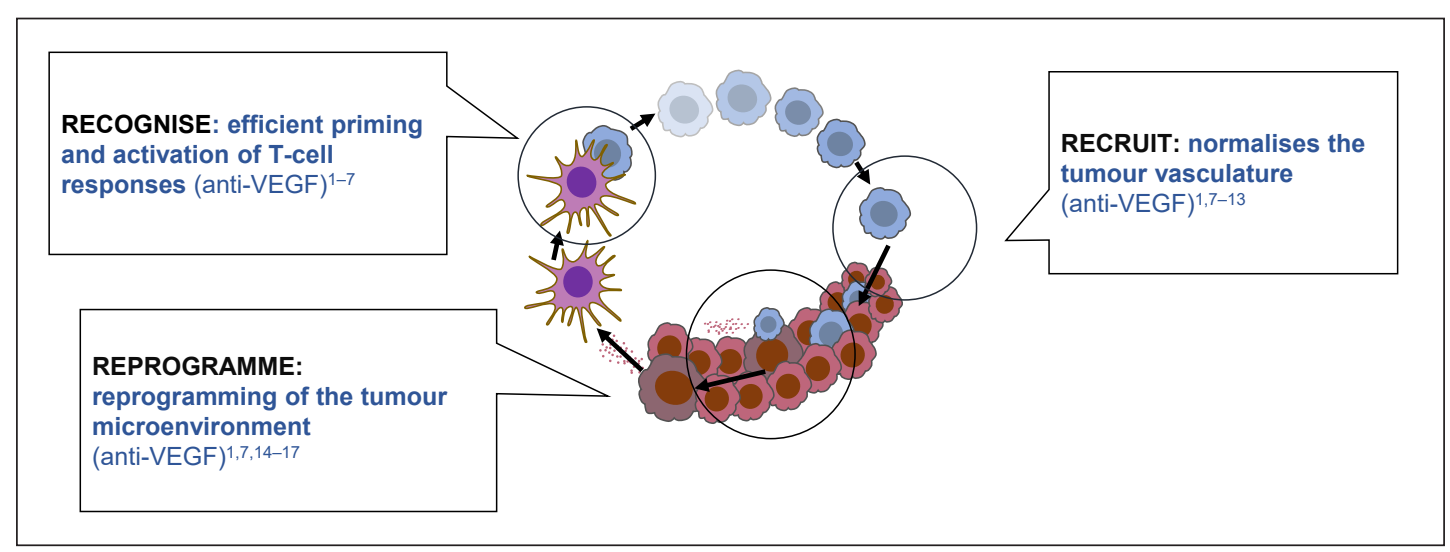

Fig. 5. Targeting VEGF influences the tumor microenvironment, T-cell priming, and trafficking/infiltration of T-cells [modified from 4, 6-8, 10-25].

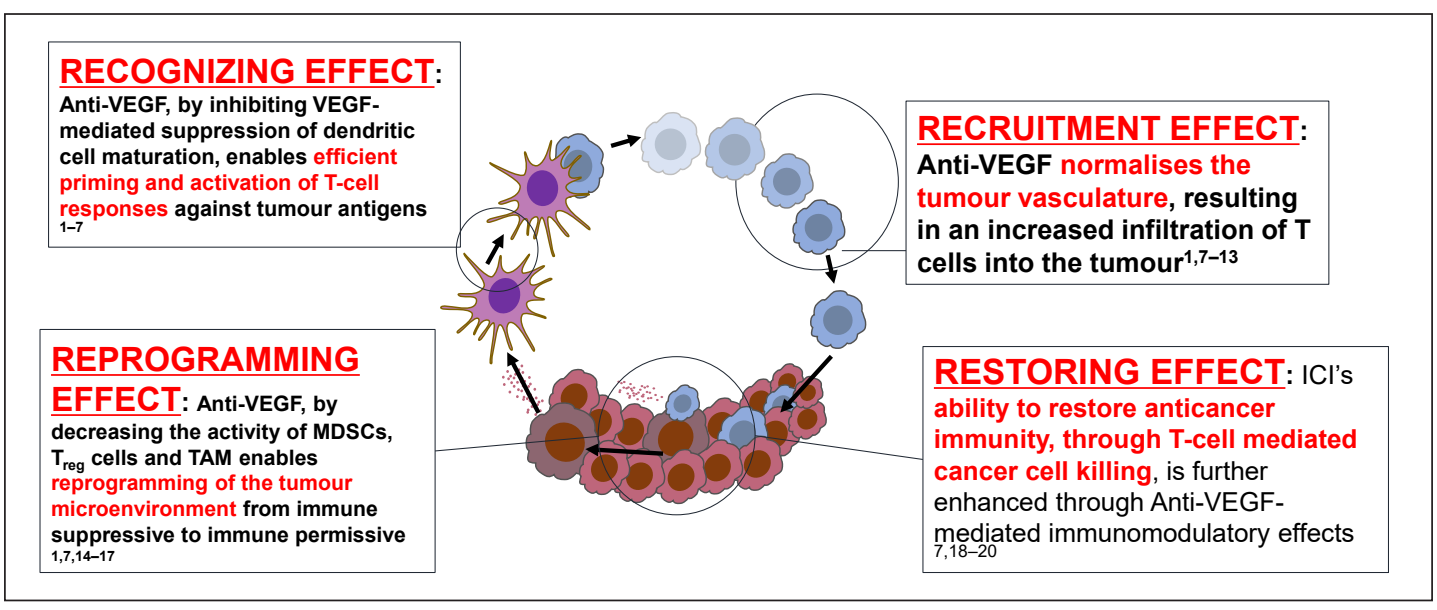

Fig. 6. Scientific rationale of ICI plus anti-VEGF antibody. This combination therapy increases Recognizing, Recruitment, Reprogramming, and Restoring effects (4Rs) in the cancer immunity cycle [modified from 4, 6-8, 10-25].

tigen peptides released by necrotic cancer cells, and comprises seven steps: (1) release of cancer cell antigens, (2) cancer antigen presentation by DCs, (3) priming and activation of T-cells, (4) trafficking of T-cells to tumors, (5) infiltration of T-cells into tumors, (6) recognition of cancer cells by T-cells, and (7) killing of cancer cells (Fig. 3). VEGF disrupts this cancer-immunity cycle by inducing immune escape through the mechanisms described earlier at almost every step of the cycle [4-8, 10-21, 25, 26] (Fig. 4). In the liver, hepatic interstitial cells (e.g., Kupffer cells, sinusoidal endothelial cells, and hepatic stellate cells) are also thought to be components of the immunosuppressive microenvironment [27]. Based on this theoretical background, anti-VEGF therapies such as anti-VEGF antibodies and multikinase inhibitors that possess VEGF receptor inhibitory activity will (1) enhance the antigen presentation ability of DCs, (2) promote T-cell activation in the priming phase, (3) normalize tumor vasculature and thereby improve trafficking and infiltration of T-cells from the lymph nodes to the tumor, (4) convert an immunosuppressive tumor microenvironment into an immune-permissive one by downregulating Tregs, TAMs, and MDSCs (described earlier), thereby negatively regulating humoral factors such as TGF- $\beta$ and IL-10 [1-17] (Fig. 5), and, at this point, (5) facilitate the effect of anti-PD-1/PD-L1 therapy to enhance antitumor activity (Fig. 6). The combination of anti-VEGF and anti-PD-1/ 


\section{Liver
Cancer}

\begin{tabular}{|c|c|}
\hline \multicolumn{2}{|c|}{ Liver Cancer 2020;9:119-137 } \\
\hline DOI: $10.1159 / 000505189$ & $\begin{array}{l}\text { (c) } 2020 \text { The Author(s). Published by S. Karger AG, Basel } \\
\text { www.karger.com/lic }\end{array}$ \\
\hline
\end{tabular}

Kudo: Atezolizumab plus Bevacizumab Combination Therapy

PD-L1 antibodies thus exerts the following four actions (4Rs) to restore an effective cancerimmunity cycle to attack the tumor (Fig. 6): (1) Recognizing effect: anti-VEGF inhibits VEGFmediated suppression of DC maturation, thereby enabling efficient priming and activation of T-cell responses against tumor antigens [1-7]. (2) Recruitment effect: anti-VEGF normalizes tumor vasculature, resulting in increased infiltration of T-cells into the tumor [1, 7-13]. (3) Reprogramming effect: anti-VEGF decreases the activity of MDSCs, Tregs, and TAMs, thereby enabling reprogramming of the tumor microenvironment from immunosuppressive to immunepermissive [1, 7, 14-17]. (4) Restoring effect: the ability of immune checkpoint inhibitors (ICIs) to restore anticancer immunity through T-cell-mediated cancer cell killing is further enhanced by anti-VEGF-mediated immunomodulatory effects [7,18-20]. This results in the fragmentation of proteins in necrotic tumor cells and the subsequent generation of cancer antigenic peptides. These antigens are recognized by DCs and presented as antigens by MHC class I molecules, further promoting the cancer-immunity cycle. In other words, the release of suppression imposed by VEGF normalizes the cancer-immunity cycle, leading to effective tumor necrosis through the effects of activated T-cells [4-25] (Fig. 6). Nonclinical studies of lenvatinib, a tyrosine kinase inhibitor (TKI), indicate that inhibition of the immunosuppressive tumor microenvironment involving TAMs and Tregs results in decreased TGF- $\beta$ and IL-10, downregulation of PD- 1 and Tim 3 expression, as well as induction of antitumor immunity (e.g., induction of immunostimulatory IL-12) [28-31]. These findings also serve as a rationale for studies on the combination of a TKI with VEGF receptor inhibitory activity and anti-PD-1/PD-L1 antibodies. It is also highly likely that the immunoregulatory action of TKIs is attributable to the anti-VEGF effect.

The results of a phase $1 \mathrm{~b}$ study on the combination of atezolizumab (Atezo) (an anti-PDL1 antibody) and bevacizumab (Beva) (an anti-VEGF antibody) for the treatment of advanced hepatocellular carcinoma (HCC) were reported at the American Society of Clinical Oncology (ASCO) annual meeting in June 2018. An amazingly high objective response rate (ORR) of 65\% $(15 / 23)$ by the independent review facility (IRF) per RECIST v1.1 surprised many researchers [32]. However, 4 months later at the European Society for Medical Oncology (ESMO) annual meeting in October 2018, the updated ORR obtained with a larger cohort and longer follow-up period was a disappointing ORR of $27 \%$ (20/73) [33]. The decrease in ORR was speculated to be attributed to an increase in the proportion of highly advanced HCC patients with macrovascular invasion (MVI) and/or extrahepatic spread (EHS) from 65\% (15/23) at the 2018 ASCO annual meeting to $88 \%(64 / 73)$ at the 2018 ESMO annual meeting. Indeed, the ORR was lower in advanced-stage HCC $(25 \%, 16 / 64)$, defined as positive for MVI and/or EHS, than in intermediate-stage HCC $(50 \%, 4 / 8)$, defined as both MVI- and EHS-negative. A closer look at the data reported at the 2018 ASCO annual meeting revealed that the ORR by the IRF per RECIST v1.1 was 73\% (11/15) in advanced-stage HCC (MVI and/or EHS positive) and 75\% (3/4) in intermediate-stage HCC (MVI- and EHS-negative); the ORR for advanced-stage HCC reported at the 2018 ASCO annual meeting was unusually high compared with that for intermediatestage HCC [31]. In addition, the ORR of $25 \%$ in patients with advanced-stage HCC reported at the 2018 ESMO annual meeting is likely to be reasonable, whereas that reported at the 2018 ASCO annual meeting (73\%) may have been unusually high by chance because the ORR is usually higher in intermediate- than in advanced-stage HCC, as shown in the REFLECT trial, which tested lenvatinib [34, 35]. The ORR in the entire population of the REFLECT trial was $41 \%$ per mRECIST [34], whereas a Japanese subanalysis showed a markedly higher ORR in intermediate-stage HCC (61\%) than in advanced-stage HCC (38\%) [35].

An increase in the proportion of patients with advanced HCC certainly contributed to the lower ORR reported at the 2018 ESMO annual meeting; nonetheless, the extremely high ORR in advanced-stage HCC, as well as that in intermediate-stage HCC reported at the 2018 ASCO annual meeting, were probably due to bias associated with the small sample size. Such bias can occur especially in the early stage of a single-arm study when the sample size is too small 


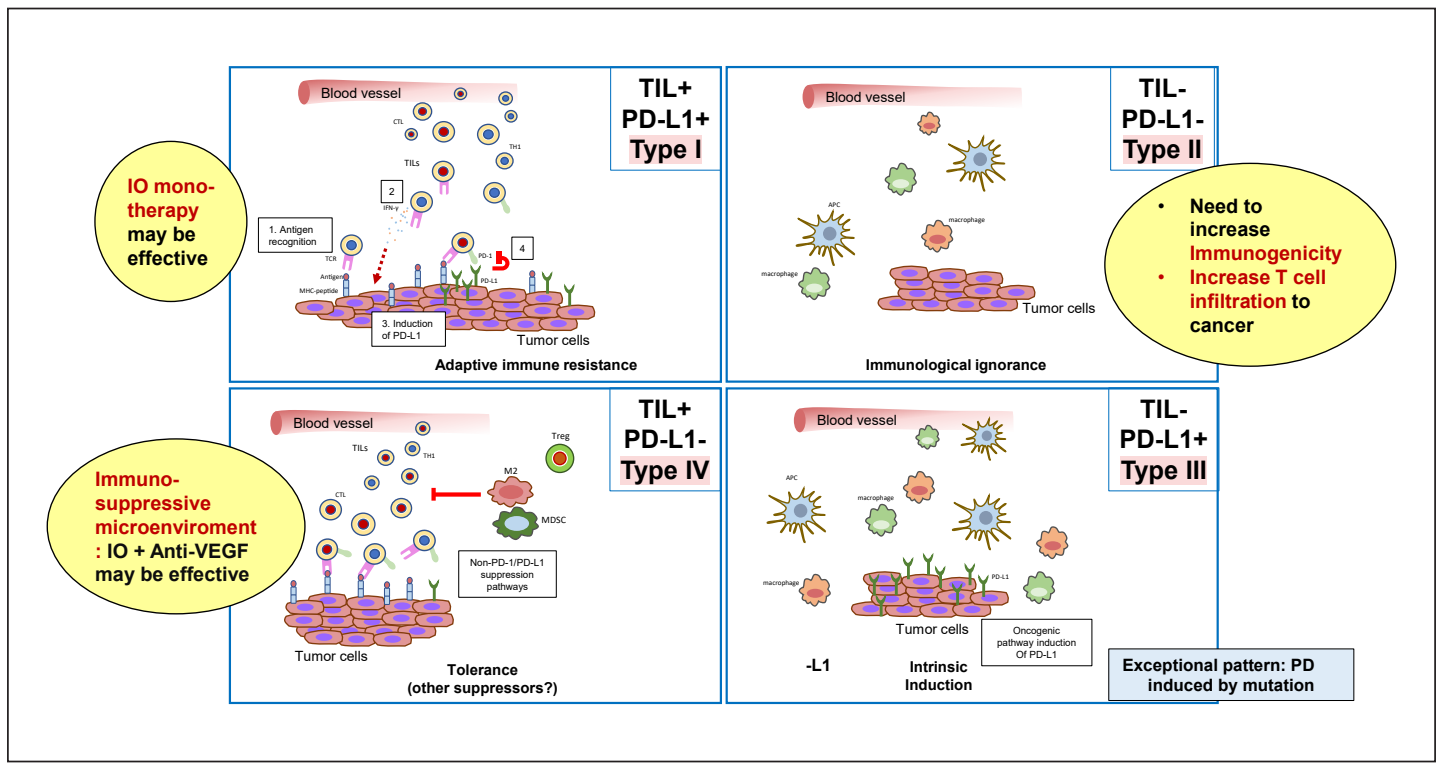

Fig. 7. Cancer is classified into four types depending on immune microenvironment (TIL: CD8+ cell and PDL1 expression) (types I-IV) [modified from 36]. ICI monotherapy is effective in type I cancer microenvironment, but not in type IV. ICI plus anti-VEGF will be effective in type IV cancer microenvironment.

$[31,33]$. This suggests that the data of single-arm studies should be interpreted with caution when the sample size is small and the duration of the observation period is short [31].

\section{Classification of the Tumor Microenvironment according to the Expression Status of PD-L1 and Tumor-Infiltrating Lymphocytes, and Immunotherapeutic Strategies}

The cancer microenvironment can be classified into four types (types I-IV) according to the presence of tumor-infiltrating lymphocytes (CD8-positive cells) and the status of PD-L1 expression [36] (Fig. 7). Type I, characterized by a positive PD-L1 status with presence of tumor-infiltrating lymphocytes, is a typical cancer microenvironment that is expected to respond adequately to monotherapy with ICIs. By contrast, type IV, characterized by negative PD-L1 status with presence of tumor-infiltrating lymphocytes, represents a cancer that does not respond to monotherapy with ICIs because the immunosuppressive tumor microenvironment inhibits the proliferation and activity of CD8-positive cells. When lymphocytes infiltrate into tumors, cancers are attacked by perforin and granzyme released by CD8-positive cells during the immune response, at least at the initial phase. This response leads to a release of IFN $\gamma$ from T-cells, and the subsequent IFN $\gamma$-IFN $\gamma$ receptor interaction induces PD-L1 expression on the cancer cell surface via the JAK-STAT signaling pathway. This enables cancer cells to evade attack from CD8-positive cells (immune escape). This is the microenvironment of type I cancer, and thus PD-1/PD-L1 antibody monotherapy is expected to be effective to overcome the immune escape. However, in type IV cancer, the PD-L1-negative status in the presence of CD8-positive cells indicates that the immune response has not occurred in the tumor microenvironment. The function of CD8-positive cells is impeded in this tumor microenvironment, and since cancer cells have not undergone attack by CD8-positive cells, there is no IFN $\gamma$-induced PD-L1 expression on the cell surface. Anti-PD-1/PD-L1 monotherapy will be ineffective in this microenvironment because immune escape via the PD-1-PD-L1 axis has not been established. In this 


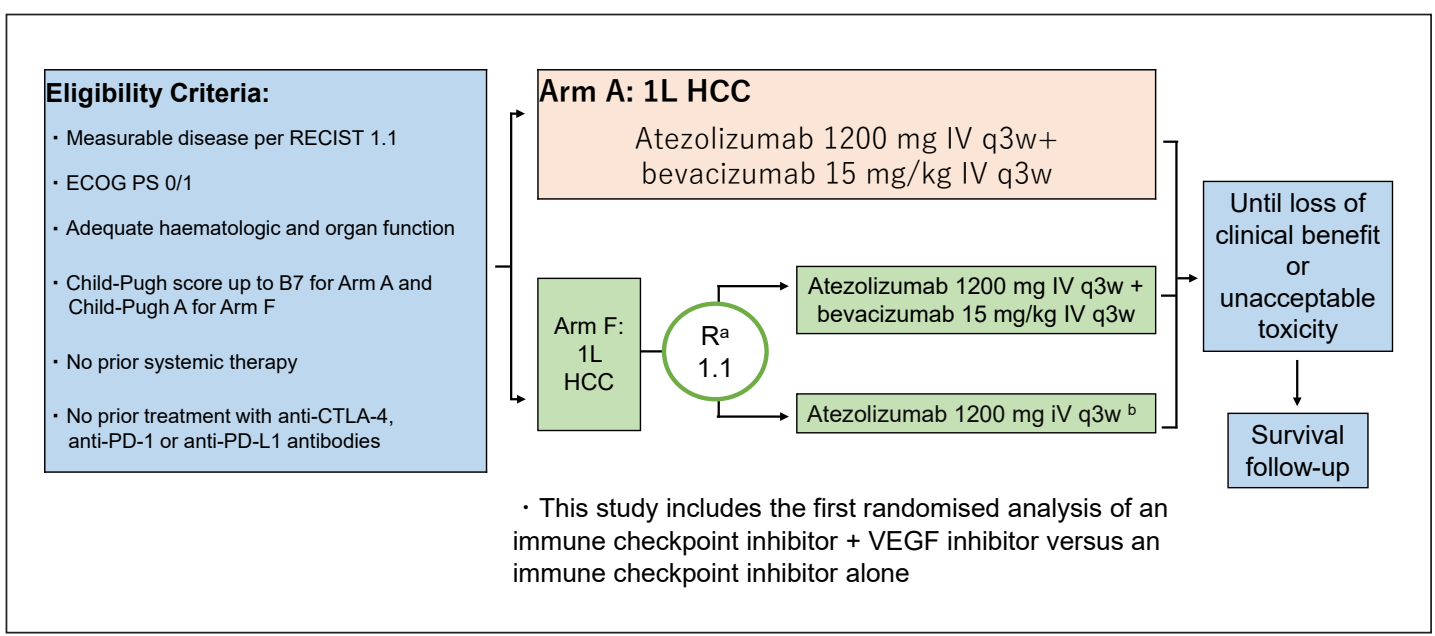

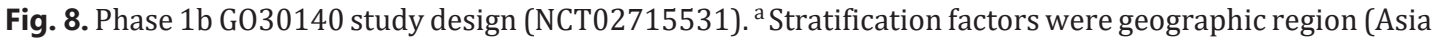
excluding Japan vs. rest of the world), MVI and/or EHS (presence vs. absence), and AFP level ( $<400$ vs. $\geq 400 \mathrm{ng} / \mathrm{mL}$ ). ${ }^{\mathrm{b}}$ Crossover was permitted.

Table 1. Phase 1b G030140 study: objectives and endpoints

\begin{tabular}{lll}
\hline & Arm A & Arm F \\
\hline Objective & $\begin{array}{l}\text { Assess the safety and efficacy of Atezo plus } \\
\text { Beva in patients with unresectable HCC }\end{array}$ & $\begin{array}{l}\text { Assess the safety and efficacy of Atezo plus } \\
\text { Beva versus Atezo monotherapy in patients } \\
\text { with unresectable HCC }\end{array}$ \\
\hline $\begin{array}{ll}\text { Primary } \\
\text { endpoints }\end{array}$ & $\begin{array}{l}\text { - ORR per IRF-assessed RECIST v1.1 } \\
\text { Key }\end{array}$ & $\begin{array}{l}\text { PFS per IRF-assessed RECIST v1.1 } \\
\text { - Safety }\end{array}$ \\
endpoints & $\begin{array}{l}\text { HCC mRECIST } \\
\text { e overall survival }\end{array}$ & \\
\hline
\end{tabular}

Median duration of follow-up: 12.4 months for arm A and 6.6 months for arm F. Atezo, atezolizumab; Beva, bevacizumab; HCC, hepatocellular carcinoma; INV, investigators; IRF, independent review facility; ORR, objective response rate; PFS, progression-free survival.

microenvironment, effective agents are those such as anti-VEGF antibodies that inhibit VEGF-induced upregulation of immunosuppressive cells (Tregs, TAMs, and MDSCs). This converts the tumor microenvironment from immunosuppressive to immune-permissive, enabling T-cells to attack cancer through antigen recognition (Fig. 5) [26]. As a result of the immune response, perforin and granzyme released by CD8-positive cells attack cancer cells, and IFN $\gamma$ induces PD-L1 expression in cancer cells. This provides the ICI (PD-1/PD-L1 antibody) with a real target (immune escape via the PD-1-PD-L1 axis) and thereby becomes an effective antitumor therapy. It is therefore logical to expect outstanding effects of antiPD-1/PD-L1 antibody plus anti-VEGF antibody/TKI combination therapy in the treatment of type IV cancer (Figs. 6, 7) [26, 36]. For type II and III cancers (cold tumors), which are characterized by the absence of tumor-infiltrating lymphocytes, different strategies such as enhancement of immunogenicity may be necessary. 


\section{Liver Cancer}

Table 2. Phase $1 \mathrm{~b}$ G030140 study (arm A): baseline characteristics [modified from 37]

\begin{tabular}{|c|c|}
\hline \multicolumn{2}{|l|}{ Liver Cancer 2020;9:119-137 } \\
\hline DOI: $10.1159 / 000505189$ & $\begin{array}{l}\text { (c) } 2020 \text { The Author(s). Published by S. Karger AG, Basel } \\
\text { www.karger.com/lic }\end{array}$ \\
\hline
\end{tabular}

Kudo: Atezolizumab plus Bevacizumab Combination Therapy

\begin{tabular}{ll}
\hline Characteristic & $\begin{array}{l}\text { Arm A: Atezo + Beva } \\
(n=104)\end{array}$ \\
\hline $\begin{array}{l}\text { Age, years } \\
\text { Male sex }\end{array}$ & $62(23-82)$ \\
Region & $84(81 \%)$ \\
$\quad$ Asia (excluding Japan) & $59(57 \%)$ \\
$\quad$ Rest of the world & $45(43 \%)$ \\
ECOG PS 1 & $52(50 \%)$ \\
Child-Pugh class & $77(74 \%)$ \\
$\quad$ A5 & $21(20 \%)$ \\
$\quad$ A6 & $6(6 \%)$ \\
B7 & \\
Etiology of HCC & $51(49 \%)$ \\
$\quad$ HBV & $31(30 \%)$ \\
$\quad$ HCV & $22(21 \%)$ \\
Nonviral & $37(36 \%)$ \\
AFP $\geq 400$ ng/mL & $74(71 \%)$ \\
EHS & $55(53 \%)$ \\
MVI & $91(88 \%)$ \\
EHS and/or MVI &
\end{tabular}

Figures are presented as median (range) or $n(\%)$. Atezo, atezolizumab; Beva, bevacizumab; ECOG PS, Eastern Cooperative Oncology Group performance status; HCC, hepatocellular carcinoma; AFP, alphafetoprotein; EHS, extrahepatic spread; MVI, macrovascular invasion.

Table 3. Phase 1b G030140 study (arm A): objective response and durability of response [modified from 37]

\begin{tabular}{llll}
\hline & \multicolumn{2}{l}{ Arm A: Atezo + Beva $(n=104)$} \\
\cline { 2 - 4 } & IRF RECIST v1.1 & IRF HCC mRECIST & INV RECIST v1.1 \\
\hline Confirmed ORR & $37(36 \%)(26-46)$ & $41(39 \%)(30-50)$ & $34(33 \%)(24-43)$ \\
CR & $12(12 \%)$ & $16(15 \%)$ & $3(3 \%)$ \\
PR & $25(24 \%)$ & $25(24 \%)$ & $31(30 \%)$ \\
DCR & $74(71 \%)$ & $74(71 \%)$ & $78(75 \%)$ \\
Ongoing response & $28(76 \%)$ & $28(68 \%)$ & $24(71 \%)$ \\
Median DOR, months & NE $(11.8$ to NE) & NE $(11.8$ to NE) & NE $(11.7$ to NE) \\
DOR range, months & $>1.6$ to $>31.0$ & $>1.6$ to $>31.0$ & $>3.5$ to $>31.0$ \\
$\quad \geq 9$ months & $20(54 \%)$ & $25(61 \%)$ & $21(62 \%)$ \\
$\quad 12$ months & $11(30 \%)$ & $11(27 \%)$ & $12(35 \%)$
\end{tabular}

Median duration of follow-up: 12.4 months. Figures are presented as $n(\%)$ or $n(\%)$ (95\% CI). Atezo, atezolizumab; Beva, bevacizumab; IRF, independent review facility; HCC, hepatocellular carcinoma; INV, investigators; NE, not estimable; ORR, objective response rate; $\mathrm{CR}$, complete response; PR, partial response.

\section{Phase 1b G030140 Study of Atezo plus Beva Combination Immunotherapy}

\section{Phase $1 b$ Single-Arm Cohort (Arm A)}

Arm A of the phase 1b G030140 study tested the combination of Atezo and Beva in patients with unresectable HCC (Fig. 8; Table 1). The different combination strategy was tested in a different cancer patient population in other arms of the phase 1b G030140 study, including patients with gastric cancer in arm B (FOLFOX + Atezo + Beva), those with pancreatic 


\section{Liver Cancer}

Table 4. Atezo plus Beva: updated results of ORR (phase $1 \mathrm{~b}$ study: $\operatorname{arm}$ A)

\begin{tabular}{|c|c|}
\hline \multicolumn{2}{|c|}{ Liver Cancer 2020;9:119-137 } \\
\hline DOI: $10.1159 / 000505189$ & $\begin{array}{l}\text { (c) } 2020 \text { The Author(s). Published by S. Karger AG, Basel } \\
\text { www.karger.com/lic }\end{array}$ \\
\hline
\end{tabular}

Kudo: Atezolizumab plus Bevacizumab Combination Therapy

\begin{tabular}{|c|c|c|c|}
\hline & $\begin{array}{l}\text { ASCO } 2018 \\
\text { Atezo + Beva } \\
(n=23)^{\mathrm{a}}\end{array}$ & $\begin{array}{l}\text { ESMO } 2018 \\
\text { Atezo + Beva } \\
(n=73)^{\mathrm{a}}\end{array}$ & $\begin{array}{l}\text { ESMO } 2019 \\
\text { Atezo + Beva } \\
(n=104)^{\mathrm{a}}\end{array}$ \\
\hline ORR & 15 (65\%) & $20(27 \%)$ & 37 (36\%) \\
\hline CR & $1(4 \%)$ & $4(5 \%)$ & $12(12 \%)$ \\
\hline PR & $14(61 \%)$ & $16(22 \%)$ & $25(24 \%)$ \\
\hline SD & $7(30 \%)$ & $35(48 \%)$ & 37 (36\%) \\
\hline PD & $1(4 \%)$ & $14(19 \%)$ & $24(23 \%)$ \\
\hline $\mathrm{DCR}^{\mathrm{b}}$ & $22(96 \%)$ & $55(75 \%)$ & 78 (75\%) \\
\hline
\end{tabular}

Figures are presented as $n(\%)$. Atezo, atezolizumab; Beva, bevacizumab; ASCO, American Society of Clinical Oncology; ESMO, European Society for Medical Oncology; ORR, objective response rate; CR, complete response; $\mathrm{PR}$, partial response; $\mathrm{SD}$, stable disease; $\mathrm{PD}$, progressive disease; DCR, disease control rate. ${ }^{a}$ Independent review facilityassessed per RECIST v1.1. ${ }^{\mathrm{b}} \mathrm{CR}+\mathrm{PR}+\mathrm{SD}$.

Table 5. Phase 1b study (arm A, $n=104$ ): PFS and OS [modified from 37]

\begin{tabular}{llll}
\hline & IRF RECIST v1.1 & IRF HCC mRECIST & INV RECIST v1.1 \\
\hline PFS & & & \\
$\quad$ Events & $69(66 \%)$ & $69(66 \%)$ & $75(72 \%)$ \\
$\quad$ PFS, months & $7.3(5.4-9.9)$ & $7.3(5.4-9.9)$ & $7.4(5.6-10.7)$ \\
6-month PFS rate & $54 \%$ & $55 \%$ & $56 \%$ \\
12-month PFS rate & $35 \%$ & $35 \%$ & $38 \%$ \\
OS & & $47(45 \%)$ & \\
Deaths & $17.1(13.8-\mathrm{NE})$ & \\
OS, months & $82 \%$ & \\
6-month OS rate & $63 \%$ & \\
12-month OS rate & & \\
\hline
\end{tabular}

Median duration of follow-up: 12.4 months. Figures are presented as median $(95 \% \mathrm{CI})$ or $n(\%)$. IRF, independent review facility; HCC, hepatocellular carcinoma; INV, investigators; PFS, progression-free survival; OS, overall survival; NE, not estimable.

cancer in arm C (gemcitabine + nab-paclitaxel + Atezo), and those with esophageal cancer in arm E (FOLFOX + Atezo or cisplatin + 5-FU + Atezo). Updated and long-term follow-up results of arm A involving 104 patients with unresectable HCC were reported at the 2019 ESMO annual meeting in Barcelona (September 27 to October 1, 2019) [37]. The majority of the subjects had highly advanced HCC; 53\% had MVI, and 88\% had EHS and/or MVI (Table 2); however, the ORR of $36 \%$ (95\% CI 26-46\%) by the IRF per RECIST v1.1 was much improved compared with the ORR of $27 \%$ reported at the 2018 ESMO annual meeting (Table 3). In addition, the ORR per mRECIST was 39\%, and the ORR by an investigator per RECIST v1.1 was $33 \%$. The complete response (CR) by the IRF per RECIST v1.1 was as high as $12 \%$ $(12 / 104)$, the partial response was $24 \%$, and the disease control rate was $71 \%$. As shown in Table 3, the response was durable: the median duration of response (DOR) was not estimable (NE) (95\% CI 11.8 months to NE); the DOR was $\geq 9$ months in 20 patients (54\%) and $\geq 12$ months in 11 patients (30\%). As stated earlier, the ORR in arm A was extremely high $(65 \%$ in 23 patients), as reported at the 2018 ASCO annual meeting, although it dropped to $27 \%$ in a larger cohort (73 patients), as reported at the 2018 ESMO annual meeting [33]. The ORR of 


\section{Liver Cancer}

Table 6. Phase $1 \mathrm{~b}$ G030140 study (arm F, $n=119)$ : baseline characteristics [modified from 37]
Table 7. Phase 1b G030140 study (arm F): objective response [modified from 37]

\begin{tabular}{l|l}
\hline Liver Cancer 2020;9:119-137 \\
\hline DOI: 10.1159/000505189 & $\begin{array}{l}\text { @ 2020 The Author(s). Published by S. Karger AG, Basel } \\
\text { www.karger.com/lic }\end{array}$ \\
\hline
\end{tabular}

Kudo: Atezolizumab plus Bevacizumab Combination Therapy

\begin{tabular}{lll}
\hline Characteristic & $\begin{array}{l}\text { Atezo }+ \text { Beva } \\
(n=60)\end{array}$ & $\begin{array}{l}\text { Atezo } \\
(n=59)\end{array}$ \\
\hline $\begin{array}{l}\text { Age (range), years } \\
\text { Male sex }\end{array}$ & $\begin{array}{l}60(22-82) \\
\text { Region }\end{array}$ & $\begin{array}{l}63(23-85) \\
49(83 \%)\end{array}$ \\
$\quad$ Asia (excluding Japan) & $39(65 \%)$ & $39(66 \%)$ \\
$\quad$ Rest of the world & $21(35 \%)$ & $20(34 \%)$ \\
ECOG PS 1 & $33(55 \%)$ & $34(58 \%)$ \\
Child-Pugh class & $43(72 \%)$ & $42(71 \%)$ \\
$\quad$ A5 & $17(28 \%)$ & $17(29 \%)$ \\
$\quad$ A6 & $34(57 \%)$ & $32(54 \%)$ \\
Etiology of HCC & $11(18 \%)$ & $10(17 \%)$ \\
$\quad$ HBV & $15(25 \%)$ & $17(29 \%)$ \\
$\quad$ HCV & $18(30 \%)$ & $19(32 \%)$ \\
$\quad$ Nonviral & $40(67 \%)$ & $39(66 \%)$ \\
AFP $\geq 400$ ng/mL & $20(33 \%)$ & $25(42 \%)$ \\
EHS & $47(78 \%)$ & $50(85 \%)$ \\
MVI &
\end{tabular}

Figures are presented as median (range) or $n(\%)$. Atezo, atezolizumab; Beva, bevacizumab; ECOG PS, Eastern Cooperative Oncology Group performance status AFP, alpha-fetoprotein; EHS, extrahepatic spread; MVI, macrovascular invasion.

\begin{tabular}{lll}
\hline & \multicolumn{2}{l}{ IRF RECIST v1.1 } \\
\cline { 2 - 3 } & $\begin{array}{l}\text { Atezo }+ \text { Beva } \\
(n=60)\end{array}$ & $\begin{array}{l}\text { Atezo } \\
(n=59)\end{array}$ \\
\hline Confirmed ORR & $12(20 \%)(11-32)$ & $10(17 \%)(8-29)$ \\
CR & $1(2 \%)$ & $3(5 \%)$ \\
PR & $11(18 \%)$ & $7(12 \%)$ \\
SD & $28(47 \%)$ & $19(32 \%)$ \\
Non-CR/non-PD & 0 & $1(2 \%)$ \\
PD & $17(28 \%)$ & $25(42 \%)$ \\
DCR & $40(67 \%)$ & $29(49 \%)$ \\
Median DOR, months & NE (NE) & NE $(3.7$ to NE) \\
\hline
\end{tabular}

Median duration of follow-up: 6.6 months. Figures are presented as $n(\%)$ or $n(\%)(95 \% \mathrm{CI})$. IRF, independent review facility; Atezo, atezolizumab; Beva, bevacizumab; ORR, objective response rate; CR, complete response; PR, partial response; SD, stable disease; PD, progressive disease; DCR, disease control rate; DOR, duration of response; $\mathrm{NE}$, not estimable. 


\section{Liver Cancer}

Table 8. Phase $1 \mathrm{~b}$ G030140 study (arm F): PFS per IRF RECIST v1.1 [modified from 37]

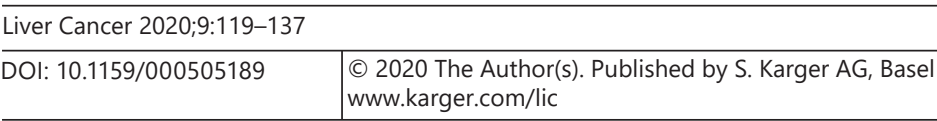

Kudo: Atezolizumab plus Bevacizumab Combination Therapy

\begin{tabular}{|c|c|c|}
\hline & $\begin{array}{l}\text { Atezo + Beva } \\
(n=60)\end{array}$ & $\begin{array}{l}\text { Atezo } \\
(n=59)\end{array}$ \\
\hline Events & $35(58 \%)$ & $39(66 \%)$ \\
\hline Median PFS, months (95\% CI) & $5.6(3.6-7.4)$ & $3.4(1.9-5.2)$ \\
\hline $\begin{array}{l}\text { Hazard ratio }(80 \% \mathrm{CI}) \\
p \text { value }\end{array}$ & \multicolumn{2}{|c|}{$\begin{array}{c}0.55(0.40-0.74) \\
0.0108\end{array}$} \\
\hline
\end{tabular}

Median duration of follow-up: 6.6 months. PFS, progression-free survival; IRF, independent review facility; Atezo, atezolizumab; Beva, bevacizumab.

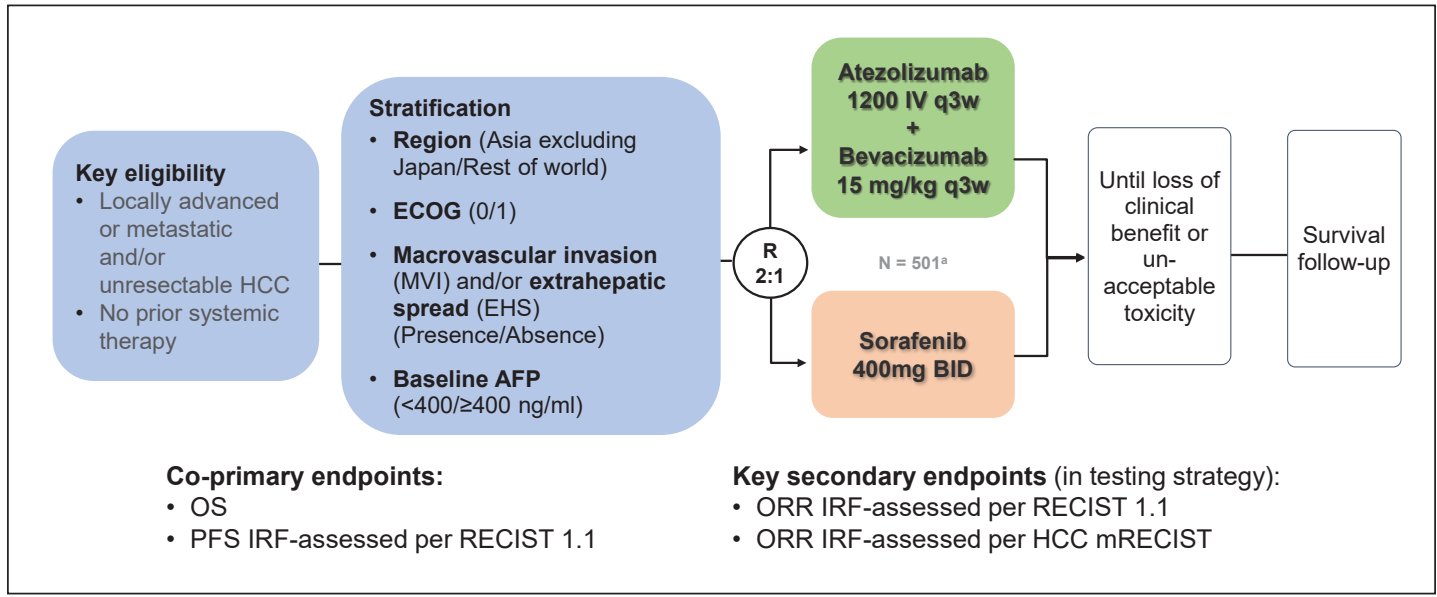

Fig. 9. Phase 3 IMbrave150 study design.

to NE); both were extremely favorable (Table 5). Given that the subjects included a considerable proportion of cases of highly advanced HCC, as mentioned earlier (MVI in 53\%, EHS and/or MVI in $88 \%$, and an alpha-fetoprotein [AFP] $\geq 400 \mathrm{ng} / \mathrm{mL}$ in $36 \%$ of subjects; Table 2), these results are extremely promising.

In arm A, 12 patients achieved CR. Surprisingly, these patients were relatively advanced-stage HCC; 4 (33\%) had AFP $\geq 400 \mathrm{ng} / \mathrm{mL}, 9$ (75\%) had EHS, 5 (42\%) had MVI, and $10(83 \%)$ had EHS and/or MVI [38]. These results suggest that advanced HCC has more chance to achieve CR than intermediate-stage HCC, which has never been reported with other approved targeted agents, and this could be an important point of immunotherapy or ICI plus anti-VEGF antibody. Conversely, another important finding in arm A is that ORR in intermediate-stage HCC was much higher $(62 \%$ [8/13]) than that in advanced-stage HCC (32\% [29/91]) [38]. This finding suggests that Atezo plus Beva combination therapy may play an important role as the first-line therapy in intermediate-stage HCC with high tumor burden, in which good results were obtained with lenvatinib-TACE sequential therapy $[39,40]$.

\section{Phase $1 b$ Randomized Cohort (Arm F)}

Arm F of the phase 1b G030140 study is a randomized study comparing PFS between patients receiving first-line combination therapy using Atezo (1,200 mg i.v.) plus Beva (15 $\mathrm{mg} / \mathrm{kg}$ ) given once every 3 weeks and those receiving monotherapy with Atezo $(1,200 \mathrm{mg}$ 
Kudo: Atezolizumab plus Bevacizumab Combination Therapy

Table 9. IMbrave150 study: baseline characteristics (ITT) [modified from 47]

\begin{tabular}{lcc}
\hline Characteristic & Atezo + Beva $(n=336)$ & Sorafenib $(n=165)$ \\
\hline Age, years & $64(26-88)$ & $66(33-87)$ \\
Male sex & $277(82 \%)$ & $137(83 \%)$ \\
Region & $133(40 \%)$ & $68(41 \%)$ \\
$\quad$ Asia (excluding Japan) & $203(60 \%)$ & $97(59 \%)$ \\
$\quad$ Rest of the world & $127(38 \%)$ & $62(38 \%)$ \\
ECOG PS 1 & $333(99 \%)$ & $165(100 \%)$ \\
Child-Pugh class & $1(<1 \%)$ & 0 \\
$\quad$ A & & \\
B & $164(49 \%)$ & $76(46 \%)$ \\
Etiology of HCC & $72(21 \%)$ & $36(22 \%)$ \\
$\quad$ HBV & $100(30 \%)$ & $53(32 \%)$ \\
$\quad$ HCV & $8(2 \%)$ & $6(4 \%)$ \\
$\quad$ Nonviral & $52(15 \%)$ & $26(16 \%)$ \\
BCLC staging at study entry & $276(82 \%)$ & $61(37 \%)$ \\
A & $126(38 \%)$ & $93(56 \%)$ \\
B & $212(63 \%)$ & $71(43 \%)$ \\
C & $129(38 \%)$ & $120(73 \%)$ \\
AFP $\geq 400$ ng/mL & $258(77 \%)$ & \\
EHS & & \\
MVI & & \\
EHS and/or MVI & & \\
\hline
\end{tabular}

Figures are presented as median (range) or $n$ (\%). ITT, intention to treat; Atezo, atezolizumab; Beva, bevacizumab; ECOG PS, Eastern Cooperative Oncology Group performance status; HCC, hepatocellular carcinoma; BCLC, Barcelona Clinic Liver Cancer; AFP, alpha-fetoprotein; EHS, extrahepatic spread; MVI, macrovascular invasion.

i.v.) (Fig. 8; Table 1). This is an important proof-of-concept study that evaluates whether the favorable results of arm A are solely due to the efficacy of Atezo or specifically due to the combination strategy of Atezo with Beva (Atezo + Beva). As shown in Table 6, subjects in the Atezo + Beva group included those with poor prognosis (MVI in 33\% and EHS and/or MVI in $78 \%$ of subjects). The relatively short observation period (6.6 months) may have contributed to the relatively low ORR (by the IRF per RECIST v1.1) in the Atezo + Beva group $(20 \%)$ as compared with that in arm A (Table 7). The ORR in the combination group should get better with a longer observation period because of the late responders as observed in arm A. In addition, the ORR in the Atezo + Beva group was slightly higher (20\%) than that achieved by conventional anti-PD-1/PD-L1 monotherapy (15-18.4\%) with nivolumab or pembrolizumab, which is similar to that of $17 \%$ with atezolizumab (Table 7) [41-45]. The effect of the addition of Beva to Atezo monotherapy was obvious: the median PFS was 5.6 months (95\% CI 3.6-7.4) in the Atezo + Beva group and 3.4 months (95\% CI 1.9-5.2 months) in the Atezo alone group, with a HR of 0.55 (95\% CI $0.40-0.74 ; p=0.0108$; Table 8). The PFS of 5.6 months in the Atezo + Beva group in arm F is relatively short compared with that of 7.3 months in arm A; however, this may also be attributed to the shorter median observation period in arm $\mathrm{F}$ (6.6 months) than in arm A (12.4 months). Once the observation period becomes long enough, both ORR and PFS in the Atezo + Beva group in arm F should become comparable to those in arm A. Nevertheless, the results of arm F are important because they provide proof of concept that Beva changes the tumor microenvironment from immunosuppressive to immune-permissive, thereby considerably enhancing the therapeutic effect of Atezo monotherapy. 


\section{Liver Cancer}

Table 10. Efficacy results of phase 3 IMbrave150 study: OS and PFS [modified from 47]

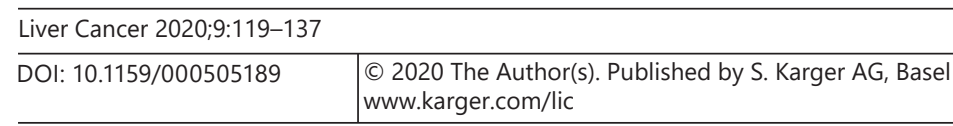

Kudo: Atezolizumab plus Bevacizumab Combination Therapy

\begin{tabular}{lcc}
\hline Co-primary endpoints & $\begin{array}{l}\text { Atezo }+ \text { Beva } \\
(n=336)\end{array}$ & $\begin{array}{l}\text { Sorafenib } \\
(n=165)\end{array}$ \\
\hline OS & NE & $13.2(10.4$ to NE $)$ \\
$\quad \begin{array}{l}\text { Median, months } \\
\text { Hazard ratio }\end{array}$ & $0.58(0.42-0.79)$ \\
$\begin{array}{l}p \text { value } \\
\text { PFS }\end{array}$ & 0.0006 \\
$\quad \begin{array}{c}\text { Median, months } \\
\text { Hazard ratio } \\
p \text { value }\end{array}$ & $6.8(5.7-8.3)$ & $4.3(4.0-5.6)$ \\
& $0.59(0.47-0.76)$ \\
\end{tabular}

Median duration of follow-up: 8.6 months. Figures in parentheses are 95\% CI. OS, overall survival; PFS, progression-free survival; Atezo, atezolizumab; Beva, bevacizumab; NE, not estimable.

\section{Phase 3 IMbrave150 Study}

The phase 3 IMbrave150 study is an international multicenter phase 3 open-label study including patients with locally advanced or metastatic and/or unresectable HCC. Patients were randomly allocated at a 2:1 ratio to the Atezo + Beva group (336 patients) or the sorafenib group, the current standard of care (165 patients). As shown in Figure 9, the co-primary endpoints were OS and PFS by the IRF per RECIST v1.1; stratification factors were geographic region (Asia excluding Japan vs. the rest of the world), Eastern Cooperative Oncology Group performance status (ECOG PS) 0:1, MVI and/or EHS (presence vs. absence), and serum AFP level ( $<400 \mathrm{ng} / \mathrm{mL}$ vs. $\geq 400 \mathrm{ng} / \mathrm{mL})$.

The phase 3 IMbrave150 study was underway (first patient-in date March 15, 2018) when results of the phase $1 \mathrm{~b}$ study were reported at the 2018 ASCO annual meeting in June 2018. Enrollment speed in this phase 3 study was unusually fast because a total of 501 patients were enrolled during 10 months, which is 6 months earlier than planned (target date July 2019 , last patient-in date January 30,2019 ). This could be related to the amazingly high ORR $(65 \%)$ in the Atezo + Beva group in arm A reported at the 2018 ASCO annual meeting. This factor may have contributed to the first interim analysis results in which both primary endpoints (OS and PFS) were met, as seen in the press release from Roche on October 21, 2019 [46]. Success in meeting OS as well as PFS endpoints with significantly good hazard ratios was beyond the expectations of many researchers, which shocked the world and indicated the arrival of a "new era of systemic therapy of HCC." Detailed results were subsequently presented at the 2019 ESMO Asia meeting held in Singapore on November 23, 2019 [47].

\section{IMbrave150 OS}

As shown in Table 9, 38\% (129/336) of subjects had MVI, 78\% (258/336) had EHS and/ or MVI, and 38\% (126/336) had AFP $\geq 400 \mathrm{ng} / \mathrm{mL}$. These proportions indicate similar, or slightly worse, prognoses compared with those in the subjects of arm $\mathrm{F}$ of the phase $1 \mathrm{~b}$ study (MVI in 33\%, MVI and/or EHS in 78\%, and AFP $\geq 400 \mathrm{ng} / \mathrm{mL}$ in 30\%; Table 6). Nevertheless, the results of the IMbrave150 study were surprisingly good; specifically, OS was NE in the Atezo + Beva group, whereas it was 13.2 months (95\% CI 10.4 months to NE) in the sorafenib group with a HR of 0.58 (95\% CI 0.42-0.79; $p=0.0006$; Table 10). An approximate OS of 22.8 months, which was derived through a primitive calculation on paper by dividing the OS of 13.2 months in the sorafenib group by the HR of 0.58 in the Atezo + Beva group, might be 
Table 11. Response rate and duration of response [modified from 47]

\begin{tabular}{|c|c|c|c|c|}
\hline & \multicolumn{2}{|l|}{ IRF RECIST v1.1 } & \multicolumn{2}{|l|}{ IRF HCC mRECIST } \\
\hline & $\begin{array}{l}\text { Atezo + Beva } \\
(n=326)\end{array}$ & $\begin{array}{l}\text { sorafenib } \\
(n=159)\end{array}$ & $\begin{array}{l}\text { Atezo + Beva } \\
(n=325)^{\mathrm{a}}\end{array}$ & $\begin{array}{l}\text { sorafenib } \\
(n=158)\end{array}$ \\
\hline Confirmed ORR, $n(\%)(95 \% \mathrm{CI})$ & $89(27)(23-33)$ & $19(12)(7-18)$ & $108(33)(28-39)$ & $21(13)(8-20)$ \\
\hline $\mathrm{CR}$ & $18(6)$ & 0 & $33(10)$ & $3(2)$ \\
\hline PR & $71(22)$ & 19 (12) & $75(23)$ & $18(11)$ \\
\hline Stratified $p$ value $\mathrm{e}^{\mathrm{b}}$ & \multicolumn{2}{|c|}{$<0.0001$} & \multicolumn{2}{|c|}{$<0.0001$} \\
\hline $\mathrm{SD}, n(\%)$ & $151(46)$ & $69(43)$ & $127(39)$ & $66(42)$ \\
\hline PD, $n(\%)$ & $64(20)$ & $39(25)$ & $66(20)$ & $40(25)$ \\
\hline DCR, $n(\%)$ & $240(74)$ & $88(55)$ & $235(72)$ & $87(55)$ \\
\hline Ongoing response, $n(\%)^{\mathrm{c}}$ & 77 (87) & $13(68)$ & $84(78)$ & $13(62)$ \\
\hline Median DOR, months & $\mathrm{NE}$ & 6.3 (4.7 to $\mathrm{NE})$ & $\mathrm{NE}$ & 6.3 (4.9 to $\mathrm{NE}$ ) \\
\hline $\begin{array}{l}\text { Event-free rate at } 6 \text { months, } \\
n(\%)\end{array}$ & 88 & 59 & 82 & 63 \\
\hline
\end{tabular}

IRF, independent review facility; HCC, hepatocellular carcinoma; Atezo, atezolizumab; Beva, bevacizumab; ORR, objective response rate; CR, complete response; PR, partial response; SD, stable disease; PD, progressive disease; DCR, disease control rate; DOR, duration of response; NE, not estimable. ${ }^{a}$ IRF HCC mRECIST-evaluable population was based on patients who presented with measurable disease at baseline per HCC mRECIST criteria. ${ }^{b}$ Stratification factors included geographic region (Asia vs. rest of the world, including Japan), AFP level ( $<400$ vs. $\geq 400 \mathrm{ng} / \mathrm{mL}$ ) at baseline, and MVI and/or EHS (yes vs. no) per IxRS. ${ }^{c}$ Denominator is patients with confirmed CR/PR. Data cutoff, 29 August 2019; median survival follow-up, 8.6 months.

achieved. Although this calculation is not a scientifically correct way and is just speculative, it may be an easy way to picture the real impact of this combination on OS. This simply estimated OS is considerably longer than that in arm A (17.1 months). Given that a poorer prognosis was expected in arm A than in the Atezo + Beva group in the IMbrave150 study based on the proportion of subjects with MVI (53 vs. $38 \%$ ), the estimation of OS ( $\geq 20$ months) in the Atezo + Beva group seems reasonable. There might be a discussion regarding the fact that overestimation of benefit may exist in studies that stop early, especially if they actually stop at the first interim analysis when two interim analyses have been planned [48]. Also, "systematic review and meta-regression analysis suggested that ratio of risk ratio in the truncated RCTs and matching nontruncated RCTs was 0.71" [49]. Therefore, the final HR could be estimated as $0.58 / 0.71=0.82$ if the trial is continued to the final analysis. This dilution of OS is usually caused by posttrial treatment. However, it is also true that "positive is positive." This is what the clinical trial is, i.e., this statistical method is obviously guaranteed by modern biostatistics and regulatory authorities. Although there have been no agents more effective than sorafenib, this combination strategy is the first to show a clinical benefit over sorafenib. It is noteworthy that the alpha value was split to 0.0033 for OS and 0.002 for PFS in the statistical plan of the first interim analysis and was sufficiently met here in few events $(161 / 501)$ compared with the planned final events of 312. This achievement of endpoints at the first interim analysis can be explained as follows: (1) the outstanding efficacy of Atezo plus Beva combination therapy, (2) a total of 501 patients enrolled in the extremely short period of 10 months, with 161 events occurring in succession in the sorafenib group (39\% [65/165]) compared with the combination arm (29\% [96/336]), and (3) since OS was analyzed at the early stage (at first interim analysis), there was not enough time for patients in the control (sorafenib) group to benefit from subsequent therapy after progression. These patients usually benefit from post-progression treatment, especially from other types of ICIs (nivolu- 


\section{Liver Cancer}

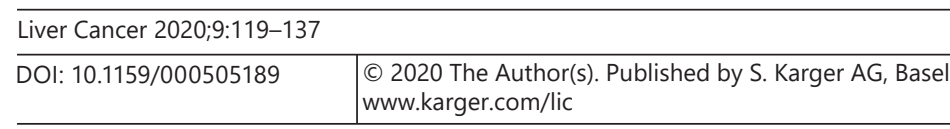

Kudo: Atezolizumab plus Bevacizumab Combination Therapy

mab or pembrolizumab, which are both approved as second-line therapies after sorafenib), even if the percentage of patients receiving immunotherapy was similar to that in CheckMate 459 after the sorafenib arm [41]. Therefore, since the median survival follow-up period was short (8.6 months), the extension of OS might have been minimally affected by the postprogression survival related to subsequent immunotherapy [50]. In fact, as reported at the 2019 ESMO annual meeting, the CheckMate 459 study did not meet its primary endpoint of OS at the first interim analysis, and extension of OS by nivolumab over control (sorafenib) was not shown even in the final analysis. This could be attributed to the long-tail effect in the sorafenib group because an ICI was administered after sorafenib in $20 \%$ of patients along with investigational drug including immunotherapy (10\%), and this could have resulted in a longer OS than expected in the sorafenib group [41]. Actually, OS in the sorafenib arm was the longest (14.7 months) in the CheckMate 459 study among the previous trials.

\section{Progression-Free Survival}

The IMbrave 150 study reported a PFS by the IRF per RECIST v1.1 of 6.8 months (95\% CI 5.7-8.3 months) in the Atezo + Beva group and 4.3 months (95\% CI 4.0-5.6 months) in the sorafenib group, with a HR of 0.59 (95\% CI 0.47-0.76\%; $p<0.0001)$. This PFS in the Atezo + Beva group was shorter than that in arm A (7.3 months), but considerably longer than that in arm F (5.6 months). Given that the median observation period in the IMbrave150 study (8.6 months) was shorter than that in phase $1 \mathrm{~b}$ arm A (12.4 months), the PFS will eventually be extended as the observation period becomes longer because of the late responders observed in phase $1 \mathrm{~b}$ arm $\mathrm{A}$.

\section{ORR and DOR}

The difference in ORR by the IRF per RECIST v1.1 was significant (stratified $p$ value $<0.0001$ ) between the Atezo + Beva group (27\%; 95\% CI 23-33\%) and the sorafenib group (12\%; 95\% CI 7-18\%). In the phase $1 \mathrm{~b}$ study, the ORR in arm A was $36 \%$ and that in arm $\mathrm{F}$ was $20 \%$. Although the ORR in the Atezo + Beva group in the IMbrave150 study was lower than that in arm A, it may be elevated as the observation period becomes longer. CR was achieved in 18 patients (6\%), which is quite promising. The median DOR of NE and the proportion of responders with a DOR $>6$ months of $80 \%$ indicate considerably durable responses to treatment (Table 11).

\section{Forest Plot (Subanalysis)}

In the IMbrave150 study, subanalyses showed better OS and PFS in the Atezo + Beva group irrespective of age, sex, region, ECOG PS, etiology, AFP level, presence or absence of MVI and/or EHS, and prior therapy. In particular, both the presence and absence of MVI/EHS were better in the combination arm. The OS and PFS for patients with viral hepatitis B or C etiology were favorable for the combination arm compared with those in patients with nonviral etiology. This was in agreement with the results obtained for nivolumab in the CheckMate 459 trial [41]. The reason for this result is unknown at present, and it should be kept in mind and clarified in the near future.

\section{Patient-Reported Outcome: Quality of Life}

The patient-reported outcome was considerably better in the Atezo + Beva group than in the sorafenib group. Specifically, median time to deterioration of quality of life was longer in the Atezo + Beva group than in the sorafenib group (11.2 vs. 3.6 months) according to the EORTC-QLC-C30 measure, with a HR of 0.63 (95\% CI $0.46-0.85 \% ; p=0.0028$ ). The only other study that demonstrated a similar favorable effect on quality of life was a REACH-2 study with ramucirumab [51] because generally, unlike antibody-based agents, TKI treatment is asso- 


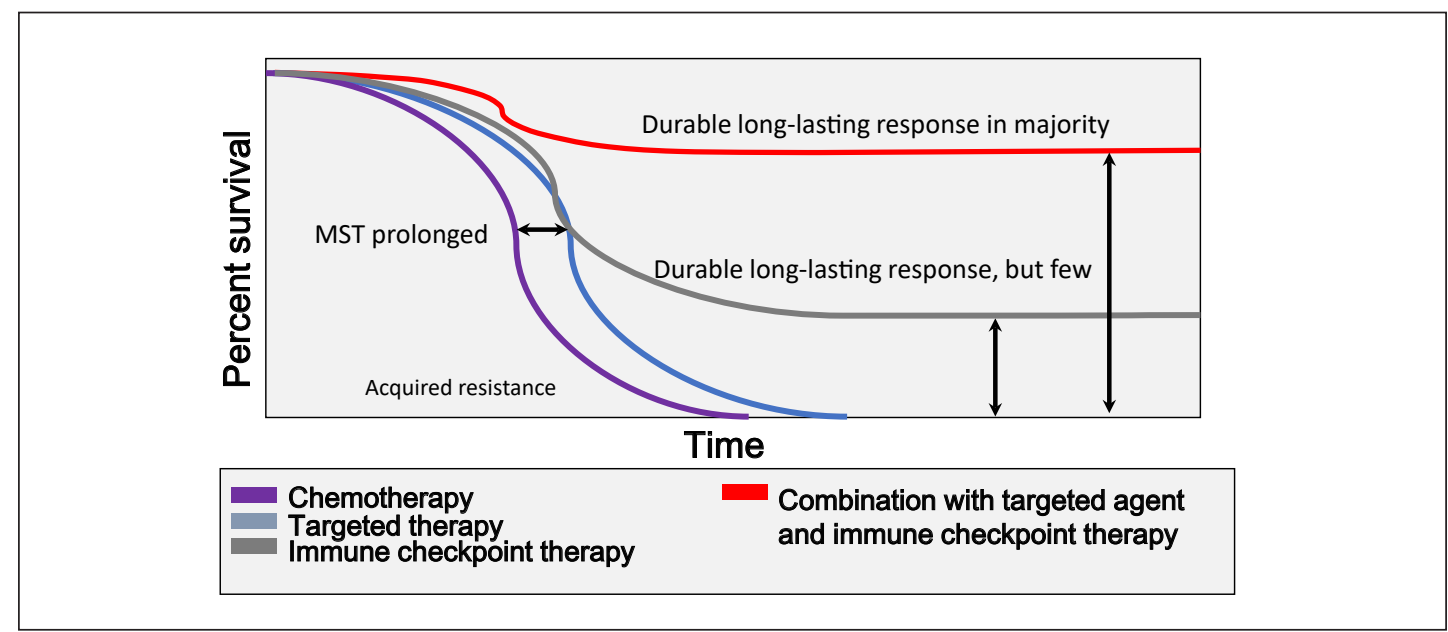

Fig. 10. Durable long-lasting response is expected in the majority of cancer patients by a combination immunotherapy with targeted agents/anti-VEGF agent and ICIs [modified from 50].

ciated with various adverse events. Indeed, the SHARP study similarly examined the time to symptomatic progression and failed to find improvement [52]. A favorable effect was achieved by delayed onset of symptoms caused by this combination of agents as well as by the inherent nature of antibody therapy in terms of low incidence of adverse events and good tolerability.

Safety

Treatment-related adverse events of grade 3 and 4 tended to occur at a higher rate in the sorafenib group (46\%, 71/156) than in the Atezo + Beva group (36\%, 117/329). Among adverse events occurring at a rate $\geq 10 \%$, those that commonly occurred in the sorafenib group, such as diarrhea, hand-foot skin reaction, decreased appetite, hypertension, alopecia, and asthenia, were less common in the Atezo + Beva group. Elevated alanine transaminase level and proteinuria were slightly more common in the Atezo + Beva group than in the sorafenib group, although these were grade 1 or 2 . Most surprisingly, almost no immune-related adverse events except for some infusion reaction were observed in the Atezo + Beva group unlike other ICIs such as nivolumab [41] or pembrolizumab [43]. In addition, Beva-related bleeding events were minimal. Taken together, the safety profile was tremendously favorable in the Atezo + Beva treated group and there were no previously unreported adverse events.

\section{Summary of the Results of the IMbrave150 Study}

The IMbrave150 study showed extremely positive results for all primary and secondary endpoints (OS, PFS, ORR, quality of life, and safety) in the comparison of Atezo + Beva over sorafenib at the first interim analysis. These unprecedented clinical results support the theory that anti-VEGF therapy converts the tumor microenvironment from immunosuppressive to immune-permissive, thereby enhancing the effect of PD-1/PD-L1 antibodies and consequently improving the antitumor effect [36]. The Atezo plus Beva combination is revolutionary because it is the first therapy with demonstrated superiority to sorafenib since the approval of sorafenib in 2007. Most importantly, the study supported the theory that the combination strategy based on immune mechanism against cancer microenvironment actually functioned as expected. This is the biggest epoch-making and practice-changing event in the history of systemic therapy against HCC since the approval of sorafenib in 2007, and it has heralded a new era in the treatment of HCC. 
Kudo: Atezolizumab plus Bevacizumab Combination Therapy

Atezo plus Beva will definitely induce a paradigm change in the management of HCC, and there is no doubt that after this successful phase 3 study, Atezo plus Beva combination therapy will be available in routine clinical practice worldwide as the first option for first-line therapy in HCC.

\section{Conclusion}

Sharma and Allison $[53,54]$ predicted in 2015 that the durable long-lasting response and long-term survival expected in a small fraction of patients in the setting of ICI monotherapy will be further improved and will benefit more patients in the setting of combination therapy with an ICI and a molecular targeted agent such as bevacizumab (Fig. 10) [53]. The IMbrave150 study clearly demonstrated the survival benefit of anti-PD-L1 and anti-VEGF combination therapy in patients with HCC as expected based on preclinical study [55]. Furthermore, the phase 3 IMbrave 150 study and the phase $1 \mathrm{~b}$ arm A study showed a high CR rate (6-12\%), suggesting the possibility of actual HCC cure with this combination therapy. In that sense, Atezo plus Beva combination immunotherapy will definitely bring a new era of systemic therapy and change the paradigm in HCC treatment.

\section{Statement of Ethics}

Not applicable.

\section{Disclosure Statement}

Lecture: Eisai, Bayer, MSD. Grant: EA Pharma, Eisai, Gilead, Takeda, Otsuka, Taiho. Advisory consulting: Eisai, Ono, MSD, BMS, Roche, Chugai.

\section{Funding Sources}

None.

\section{References}

1 Fukumura D, Kloepper J, Amoozgar Z, Duda DG, Jain RK. Enhancing cancer immunotherapy using antiangiogenics: opportunities and challenges. Nat Rev Clin Oncol. 2018 May;15(5):325-40.

2 Chouaib S, Messai Y, Couve S, Escudier B, Hasmim M, Noman MZ. Hypoxia promotes tumor growth in linking angiogenesis to immune escape. Front Immunol. 2012 Feb;3:21.

3 Voron T, Marcheteau E, Pernot S, Colussi O, Tartour E, Taieb J, et al. Control of the immune response by proangiogenic factors. Front Oncol. 2014 Apr; 4:70.

4 Chen DS, Mellman I. Oncology meets immunology: the cancer-immunity cycle. Immunity. 2013 Jul;39(1):1-10.

5 Hegde PS, Wallin JJ, Mancao C. Predictive markers of anti-VEGF and emerging role of angiogenesis inhibitors as immunotherapeutics. Semin Cancer Biol. 2018 Oct;52(Pt 2):117-24.

6 Ferrara N, Hillan KJ, Gerber HP, Novotny W. Discovery and development of bevacizumab, an anti-VEGF antibody for treating cancer. Nat Rev Drug Discov. 2004 May;3(5):391-400.

7 Gabrilovich DI, Chen HL, Girgis KR, Cunningham HT, Meny GM, Nadaf S, et al. Production of vascular endothelial growth factor by human tumors inhibits the functional maturation of dendritic cells. Nat Med. 1996 0ct; 2(10):1096-103.

8 Gabrilovich D, Ishida T, Oyama T, Ran S, Kravtsov V, Nadaf S, et al. Vascular endothelial growth factor inhibits the development of dendritic cells and dramatically affects the differentiation of multiple hematopoietic lineages in vivo. Blood. 1998 Dec;92(11):4150-66.

9 Elovic AE, Ohyama H, Sauty A, McBride J, Tsuji T, Nagai M, et al. IL-4-dependent regulation of TGF-alpha and TGF-beta1 expression in human eosinophils. J Immunol. 1998 Jun;160(12):6121-7. 
10 Guermonprez P, Valladeau J, Zitvogel L, Théry C, Amigorena S. Antigen presentation and T cell stimulation by dendritic cells. Annu Rev Immunol. 2002;20(1):621-67.

11 Villadangos JA, Schnorrer P. Intrinsic and cooperative antigen-presenting functions of dendritic-cell subsets in vivo. Nat Rev Immunol. 2007 Jul;7(7):543-55.

12 Griffioen AW, Damen CA, Blijham GH, Groenewegen G. Tumor angiogenesis is accompanied by a decreased inflammatory response of tumor-associated endothelium. Blood. 1996 Jul;88(2):667-73.

13 Griffioen AW, Damen CA, Martinotti S, Blijham GH, Groenewegen G. Endothelial intercellular adhesion molecule-1 expression is suppressed in human malignancies: the role of angiogenic factors. Cancer Res. 1996 Mar;56(5):1111-7.

14 Goel S, Duda DG, Xu L, Munn LL, Boucher Y, Fukumura D, et al. Normalization of the vasculature for treatment of cancer and other diseases. Physiol Rev. 2011 Jul;91(3):1071-121.

15 Motz GT, Santoro SP, Wang LP, Garrabrant T, Lastra RR, Hagemann IS, et al. Tumor endothelium FasL establishes a selective immune barrier promoting tolerance in tumors. Nat Med. 2014 Jun;20(6):607-15.

16 Hodi FS, Lawrence D, Lezcano C, Wu X, Zhou J, Sasada T, et al. Bevacizumab plus ipilimumab in patients with metastatic melanoma. Cancer Immunol Res. 2014 Jul;2(7):632-42.

17 Wallin JJ, Bendell JC, Funke R, Sznol M, Korski K, Jones S, et al. Atezolizumab in combination with bevacizumab enhances antigen-specific T-cell migration in metastatic renal cell carcinoma. Nat Commun. 2016 Aug;7(1): 12624.

18 Gabrilovich DI, Nagaraj S. Myeloid-derived suppressor cells as regulators of the immune system. Nat Rev Immunol. 2009 Mar;9(3):162-74.

19 Huang C, Li J, Song L, Zhang D, Tong Q, Ding M, et al. Black raspberry extracts inhibit benzo(a)pyrene diolepoxide-induced activator protein 1 activation and VEGF transcription by targeting the phosphotidylinositol 3-kinase/Akt pathway. Cancer Res. 2006 Jan;66(1):581-7.

20 Ko SY, Guo H, Barengo N, Naora H. Inhibition of ovarian cancer growth by a tumor-targeting peptide that binds eukaryotic translation initiation factor 4E. Clin Cancer Res. 2009 Jul;15(13):4336-47.

21 Kusmartsev S, Eruslanov E, Kübler H, Tseng T, Sakai Y, Su Z, et al. Oxidative stress regulates expression of VEGFR1 in myeloid cells: link to tumor-induced immune suppression in renal cell carcinoma. J Immunol. 2008 Jul;181(1):346-53.

22 Herbst RS, Soria JC, Kowanetz M, Fine GD, Hamid O, Gordon MS, et al. Predictive correlates of response to the anti-PD-L1 antibody MPDL3280A in cancer patients. Nature. 2014 Nov;515(7528):563-7.

23 Chen X, Zeng Q, Wu MX. Improved efficacy of dendritic cell-based immunotherapy by cutaneous laser illumination. Clin Cancer Res. 2012 Apr;18(8):2240-9.

24 Zou W, Chen L. Inhibitory B7-family molecules in the tumour microenvironment. Nat Rev Immunol. 2008 Jun; 8(6):467-77.

25 Oyama T, Ran S, Ishida T, Nadaf S, Kerr L, Carbone DP, et al. Vascular endothelial growth factor affects dendritic cell maturation through the inhibition of nuclear factor-kappa B activation in hemopoietic progenitor cells. J Immunol. 1998 Feb;160(3):1224-32.

26 Harding JJ, Watanabe T, El-Dika I, Nishida N, Abou-Alfa GK, Kudo M. Hepatocellular carcinoma. In: Haanen JBAG, Califono R, Lugowska I, Garassino MC, editors. ESMO handbook of immuno-oncology. Lugano, Switzerland: ESMO Press; 2018. p. 191-202.

27 Tiegs G, Lohse AW. Immune tolerance: what is unique about the liver. J Autoimmun. 2010 Feb;34(1):1-6.

28 Kato Y, Tabata K, Kimura T, Yachie-Kinoshita A, Ozawa Y, Yamada K, et al. Lenvatinib plus anti-PD-1 antibody combination treatment activates CD8+ T cells through reduction of tumor-associated macrophage and activation of the interferon pathway. PLoS One. 2019 Feb;14(2):e0212513.

29 Kudo M. Combination Cancer Immunotherapy in Hepatocellular Carcinoma. Liver Cancer. 2018 Mar;7(1):20-7.

30 Kudo M. Targeted and immune therapies for hepatocellular carcinoma: predictions for 2019 and beyond. World J Gastroenterol. 2019 Feb;25(7):789-807.

31 Kudo M. Combination Cancer Immunotherapy with Molecular Targeted Agents/Anti-CTLA-4 Antibody for Hepatocellular Carcinoma. Liver Cancer. 2019 Feb;8(1):1-11.

32 Stein S, Pishvaian MJ, Lee MS, Lee KH, Hernandez S, Kwan A. Safety and clinical activity of 1L atezolizumab + bevacizumab in a phase Ib study in hepatocellular carcinoma (HCC). J Clin Oncol. 2018;36(suppl):abstract 4074.

33 Pishvaian MJ, Lee MS, Ryoo B, Stein S, Lee K, Verret W, et al. Updated safety and clinical activity results from a phase Ib study of atezolizumab + bevacizumab in hepatocellular carcinoma (HCC). Munich, Germany: ESMO 2018, October 19-12; LBA26.

34 Kudo M, Finn RS, Qin S, Han KH, Ikeda K, Piscaglia F, et al. Lenvatinib versus sorafenib in first-line treatment of patients with unresectable hepatocellular carcinoma: a randomised phase 3 non-inferiority trial. Lancet. 2018 Mar;391(10126):1163-73.

35 Yamashita T, Kudo M, Ikeda K, Izumi N, Tateishi R, Ikeda M, et al. REFLECT - a phase 3 trial comparing efficacy and safety of lenvatinib to sorafenib for the treatment of unresectable hepatocellular carcinoma: an analysis of Japanese subset. J Gastroenterol. 2020 Jan;55(1):113-22.

36 Teng MW, Ngiow SF, Ribas A, Smyth MJ. Classifying Cancers Based on T-cell Infiltration and PD-L1. Cancer Res. 2015 Jun;75(11):2139-45.

37 Lee MS, Ryoo BY, Hsu CH, Numata K, Stein S, Verret W, et al. Randomised efficacy and safety results for atezolizumab + bevacizumab in patients with previous untreated, unresectable hepatocellular carcinoma. Barcelona: ESMO 2019, September 27-October 1. 
Kudo: Atezolizumab plus Bevacizumab Combination Therapy

38 Lee KH, Ryoo BY, Hsu CH, Numata K, Stein S, Verret W, et al. Phase Ib Study Results: Subgroup Efficacy Analysis of Atezolizumab + Bevacizumab in Patients With Previously Untreated, Unresectable Hepatocellular Carcinoma. Chicago: ILCA; 2019.

39 Kudo M, Ueshima K, Chan S, Minami T, Chishina H, Aoki T, et al. Lenvatinib as an Initial Treatment in Patients with Intermediate-Stage Hepatocellular Carcinoma Beyond Up-To-Seven Criteria and Child-Pugh A Liver Function: A Proof-Of-Concept Study. Cancers (Basel). 2019 Jul;11(8):E1084.

40 Kudo M. A New Treatment Option for Intermediate-Stage Hepatocellular Carcinoma with High Tumor Burden: Initial Lenvatinib Therapy with Subsequent Selective TACE. Liver Cancer. 2019 Oct;8(5):299-311.

41 Yau T, Park JW, Finn RS, Cheng AL, Mathurin P, Edeline J, et al. CheckMate 459: a randomized, multi-center phase 3 study of nivolumab vs sorafenib as first-line treatment in patients with advanced hepatocellular carcinoma. Barcelona: ESMO 2019; abstr 6572.

42 Zhu AX, Finn RS, Edeline J, Cattan S, Ogasawara S, Palmer D, et al.; KEYNOTE-224 investigators. Pembrolizumab in patients with advanced hepatocellular carcinoma previously treated with sorafenib (KEYNOTE224): a non-randomised, open-label phase 2 trial. Lancet Oncol. 2018 Jul;19(7):940-52.

43 Finn RS, Ryoo BY, Merle P, Kudo M, Bouattour M, Lim HY, et al. Results of KEYNOTE-240: phase 3 study of pembrolizumab (Pembro) vs best supportive care (BSC) for second line therapy in advanced hepatocellular carcinoma (HCC). J Clin Oncol. 2019;37(suppl):abstr 4004.

44 Kudo M. Pembrolizumab for the Treatment of Hepatocellular Carcinoma. Liver Cancer. 2019 May;8(3):14354.

45 Kudo M. Immune checkpoint blockade in hepatocellular carcinoma: 2017 update. Liver Cancer. 2016 Nov; 6(1):1-12.

46 Roche's Tecentriq in combination with Avastin increased overall survival and progression-free survival in people with unresectable hepatocellular carcinoma. Media release, Roche, October 2019. www.roche.com/ media/releases/med-cor-2019-10-21.htm.

47 Cheng AL, Qin S, Ikeda M, Galle PR, Ducreux M, Zhu AX, et al. IMbrave150: efficacy and safety results from a ph III study evaluating atezolizumab (atezo) + bevacizumab (bev) vs sorafenib (Sor) as first treatment (tx) for patients (pts) with unresectable hepatocellular carcinoma (HCC). Ann Oncol. 2019;30(suppl 9):LBA3.

48 Walter SD, Guyatt GH, Bassler D, Briel M, Ramsay T, Han HD. Randomised trials with provision for early stopping for benefit (or harm): the impact on the estimated treatment effect. Stat Med. 2019 Jun;38(14): 2524-43.

49 Bassler D, Briel M, Montori VM, Lane M, Glasziou P, Zhou Q, et al.; STOPIT-2 Study Group. Stopping randomized trials early for benefit and estimation of treatment effects: systematic review and meta-regression analysis. JAMA. 2010 Mar;303(12):1180-7.

50 Terashima T, Yamashita T, Takata N, Nakagawa H, Toyama T, Arai K, et al. Post-progression survival and progression-free survival in patients with advanced hepatocellular carcinoma treated by sorafenib. Hepatol Res. 2016 Jun;46(7):650-6.

51 Zhu AX, Finn RS, Galle PR, Llovet JM, Blanc JF, Okusaka T, et al. Ramucirumab as second-line treatment in patients with advanced hepatocellular carcinoma (HCC) and elevated alpha-fetoprotein (AFP) following firstline sorafenib: patient reported outcome results across two phase 3 studies (REACH-2 and REACH). Munich, Germany: ESMO 2018, October 19-23.

52 Llovet JM, Ricci S, Mazzaferro V, Hilgard P, Gane E, Blanc JF, et al.; SHARP Investigators Study Group. Sorafenib in advanced hepatocellular carcinoma. N Engl J Med. 2008 Jul;359(4):378-90.

53 Sharma P, Allison JP. Immune checkpoint targeting in cancer therapy: toward combination strategies with curative potential. Cell. 2015 Apr;161(2):205-14.

54 Sharma P, Allison JP. The future of immune checkpoint therapy. Science. 2015 Apr;348(6230):56-61.

55 Shigeta K, Datta M, Hato T, Kitahara S, Chen IX, Matsui A, et al. Dual programmed death receptor-1 and vascular endothelial growth factor receptor-2 blockade promotes vascular normalization and enhances antitumor immune responses in hepatocellular carcinoma. Hepatology. 2019 doi: 10.1002/hep.30889 [Epub ahead of print]. 